OPEN ACCESS

Edited by:

Jason R. Plemel,

University of Alberta, Canada

Reviewed by:

Qingyun $L i$,

Washington University in St. Louis,

United States

Chotima Böttcher

Charité - Universitätsmedizin

Berlin, Germany

*Correspondence:

Tuan Leng Tay

tuan.leng.tay@biologie.uni-freiburg.de

${ }^{\dagger}$ Present address:

Tuan Leng Tay,

Department of Anatomy \&

Neurobiology, Boston University

School of Medicine, Boston, MA,

United States; Department of Biology,

Boston University, Boston, MA,

United States

Specialty section:

This article was submitted to

Non-Neuronal Cells,

a section of the journal

Frontiers in Cellular Neuroscience

Received: 26 January 2021

Accepted: 17 March 2021

Published: 12 April 2021

Citation:

Bouadi O and Tay TL (2021) More Than Cell Markers: Understanding Heterogeneous Glial Responses to Implantable Neural Devices. Front. Cell. Neurosci. 15:658992. doi: 10.3389/fncel.2021.658992

\section{More Than Cell Markers: Understanding Heterogeneous Glial Responses to Implantable Neural Devices}

\author{
Ouzéna Bouadi ${ }^{1,2}$ and Tuan Leng Tay ${ }^{1,3,4 *}$ \\ ${ }^{1}$ Faculty of Biology, University of Freiburg, Freiburg, Germany, ${ }^{2}$ Faculty of Life Sciences, University of Strasbourg, \\ Strasbourg, France, ${ }^{3}$ BrainLinks-BrainTools Centre, University of Freiburg, Freiburg, Germany, ${ }^{4}$ Freiburg Institute of \\ Advanced Studies, University of Freiburg, Freiburg, Germany
}

Keywords: neural probe, microglia, astrocytes, oligodendrocytes, heterogeneity, neuroinflammation, acute brain injury, foreign body response

\section{INTRODUCTION}

Recent publicity surrounding a coin-size computer chip in a pig's brain has placed the spotlight on the field of neurointerfaces (Lewis, 2020). Implantable microelectrode arrays (MEAs), or neural probes, enable the study of brain activity and present promising treatment and therapeutic options for neurological conditions (Boehler et al., 2020). These range from motor and sensory impairments such as spinal cord injuries and hearing loss, to neuropsychiatric disorders including dementia, clinical depression and insomnia. Application-specific MEAs that, for example, record field potentials and neuronal activity have been validated in non-human primates and could help understand mechanisms underlying motor functions and epilepsy (Barz et al., 2017; Gerbella et al., 2021). Key design considerations for biocompatibility, efficacy and longevity of microelectrodes to maintain long-term neuronal recording and stimulation are highly dependent on brain tissue response (Polikov et al., 2005). The functional capacities of a biosensor depend on the number of surrounding neurons in a given radius $(50-350 \mu \mathrm{m})$ (He et al., 2020). Probe insertions generate inflammatory responses to acute tissue injuries and the introduction of foreign bodies, known as "foreign body response" (FBR). Chronic neuroprosthetic implants in rats at 16 weeks in contrast to 8 weeks have been shown to increase neuronal and dendritic loss, correlate with tau hyperphosphorylation seen in Alzheimer's disease and other tauopathies, and impede regeneration and recording of activity surrounding the device (McConnell et al., 2009). Assessments of acute proinflammatory events and chronic progression have largely centered on histological analyses of non-neuronal central nervous system (CNS) cells such as microglia, astrocytes and oligodendroglia, including their contribution to neuroinflammation and glial scars (Kozai et al., 2015; Prodanov and Delbeke, 2016). However, immunohistochemistry provides qualitative answers and rarely discriminates between heterogeneous cellular states (Wellman et al., 2019). Here we highlight developments that expand our knowledge of context-dependent heterogeneity of glia and bloodbrain barrier cells, proposing new approaches to examine the diverse contributions of nonneuronal CNS cells after probe implantation. Having a holistic understanding of multiple glial responses will advance neuroengineering that temper neuroinflammation and tissue scarring, thereby improving functional neuroprosthetic integration. 


\section{MICROGLIA AT THE BRAIN-MACHINE INTERFACE}

Microglia are myeloid cells of extra-embryonic origin that form the brain-resident macrophages (Ginhoux et al., 2010). Tissue damage triggers microglia-driven repair mechanisms including phagocytosis of cellular debris, chemotaxis, and initiation of cell death pathways through cytokine release (Prinz et al., 2019). As first responders of the CNS that potentially contribute to sustained neuroinflammation, microglial reactivity is widely assessed after microelectrode implantation to examine changes elicited by insertion injury and FBR (Kozai et al., 2012). Intracortical implantation of non-functional microelectrodes in rats has led to the elevation of oxidative stress markers (Ereifej et al., 2018). Microglia have been shown to increase acidosis and inflammation by the release of reactive oxygen species (ROS) in a controlled cortical impact (CCI) mouse model for neurotrauma (Ritzel et al., 2021). ROS can be detrimental to long-term functionality of an implanted sensor (Takmakov et al., 2015). Prolonged microglial reactivity or adherence to the electrode surface could threaten device efficacy and longevity in the recipient brain and diminish recording quality (Huang et al., 2020). Moreover, microglia secretion of proinflammatory cytokines such as tumor necrosis factor (TNF) and interleukin 1 (IL-1) may induce neurotoxic reactive astrocytes (Liddelow et al., 2017) to envelope the implant. Together with cell recruitment, glial scar formation and electrode encapsulation, proinflammatory microglia have earned the reputation of being noxious (Kozai et al., 2016). Yet depletion of microglia was shown to be unfavorable for scar formation, wound healing and survival of neurons and oligodendrocytes (Bellver-Landete et al., 2019), supporting the notion that they promote the stable integration of implanted MEAs. Microglial heterogeneity in the healthy, developing and diseased brain is very well-described (Stratoulias et al., 2019; Masuda et al., 2020), even if mammalian microglia mostly originate from a single erythromyeloid progenitor source in the embryonic yolk sac (Alliot et al., 1999; Ginhoux et al., 2013). However, prevailing studies of MEAs do not reveal the spectrum of neuroprotective or neurotoxic microglial subtypes.

Common markers for microglia and microglial reactivity, such as ionized calcium-binding adaptor molecule 1 (IBA-1), integrin alpha M (ITGAM, or CD11b) and CD68 (also ED1 ), are frequently used in immunohistochemical analysis of the brain-electrode interface as readout for tissue damage caused by implantation trauma and FBR (McConnell et al., 2009; Luan et al., 2017; Huang et al., 2020) (Figure 1). Transmembrane protein 119 (TMEM119) (Bennett et al., 2016) and purinergic receptor P2Y12 (P2RY12) (Butovsky et al., 2014) are excellent markers for homeostatic microglia, but are thus far rarely used for neurointerfaces. Neuroengineers considered the normalized intensity of microglial cell markers to proportionately represent the degree of inflammation (Lo et al., 2018). For instance, signal intensities of microglial cell markers surrounding insertion sites of explanted probes were examined at acute (1-3 days) or sub-chronic (up to 28 days) phases to evaluate the brainmachine interface (Lind et al., 2013; Wellman et al., 2019).
Studies on neurotrauma and FBR showed that microglia could upregulate proinflammatory inducible nitric oxide synthase (iNOS) (Madathil et al., 2018) or anti-inflammatory arginase 1 (Arg1) (Sawyer et al., 2014). Descriptions of microglial cell morphologies in the assessment of FBR after implantation of MEAs include "ramifying" and "amoeboid", which are, respectively, associated with steady and reactive states (Huang et al., 2020). Additional classifications such as "primed," "hypertrophic" and "hypo- or hyper-ramified" are also relevant for the phenotypic characterization of neuroprotective or neurotoxic microglia in pathological conditions (Verdonk et al., 2016). Current immunohistochemical analyses however mostly disregards microglial diversity at the implantation site.

\section{ASTROCYTES AND SCARRING AT NEUROINTERFACES}

Astrocytes are star-shaped, heterogeneous glial cells that provide significant neurotrophic support through their interaction with every component of the CNS parenchyma (Verkhratsky and Nedergaard, 2018). They support synapse formation, maturation and pruning, and modulate pre- and post-synaptic transmission in homeostatic CNS (Sofroniew and Vinters, 2010). Tissue damage unleashes reactive astrocytes that adopt neuroprotective or neurodegenerative properties (Liddelow and Barres, 2017). Glial fibrillary acidic protein (GFAP) is the most frequently used immunohistochemical marker for reactive astrocytes in analyses of brain-electrode interface and is positively correlated to astrogliosis and glial scar formation (Polikov et al., 2005; Seymour and Kipke, 2007; Kozai et al., 2015; Prodanov and Delbeke, 2016) (Figure 1). $\mathrm{GFAP}^{+}$astrocytes contribute to scarring through secretion of extracellular matrix chondroitin sulfate proteoglycans (CSPGs) such as neurocan, phosphacan and brevican (Fawcett and Asher, 1999; Matsui et al., 2002). CSPGs are inhibitors of axonal growth and remyelination that are frequently found in multiple sclerosis lesions where they reduce adherence of oligodendrocyte precursor cells (OPCs) for myelin repair (Galtrey and Fawcett, 2007; Lau et al., 2012). Inserting pieces of nitrocellulose filter into adult rat brain cortices induced infiltration of $\mathrm{GFAP}^{+}$astrocytes into the implants and continued release of CSPGs even at 1 month after tissue injury (McKeon et al., 1991, 1999). Reactive astrocytes formed the principal cell type that increasingly compacted around and encapsulated a silicon microprobe implanted for up to 12 weeks in rats (Turner et al., 1999). This was similarly observed in a marmoset brain carrying an array with 32 Teflon-coated $50-\mu \mathrm{m}$ large microelectrodes for 7 months (Budoff et al., 2019). High levels of CSPGs were concomitantly observed with neuronal loss after an uncoated silicon neural probe was implanted in rat brains (Zhong and Bellamkonda, 2007). Recording performance of multichannel, 16-shank, silicon "Utah" MEAs embedded yearlong in feline sensorimotor cortex reportedly dropped when neuronal action potentials were recorded (McCreery et al., 2016). This implicates astrocytic glial scar and neuronal death in the loss of biosensor performance. However, reactive astrocytes 


\section{IMMUNOHISTOCHEMICAL ANALYSIS OF GLIAL RESPONSE}

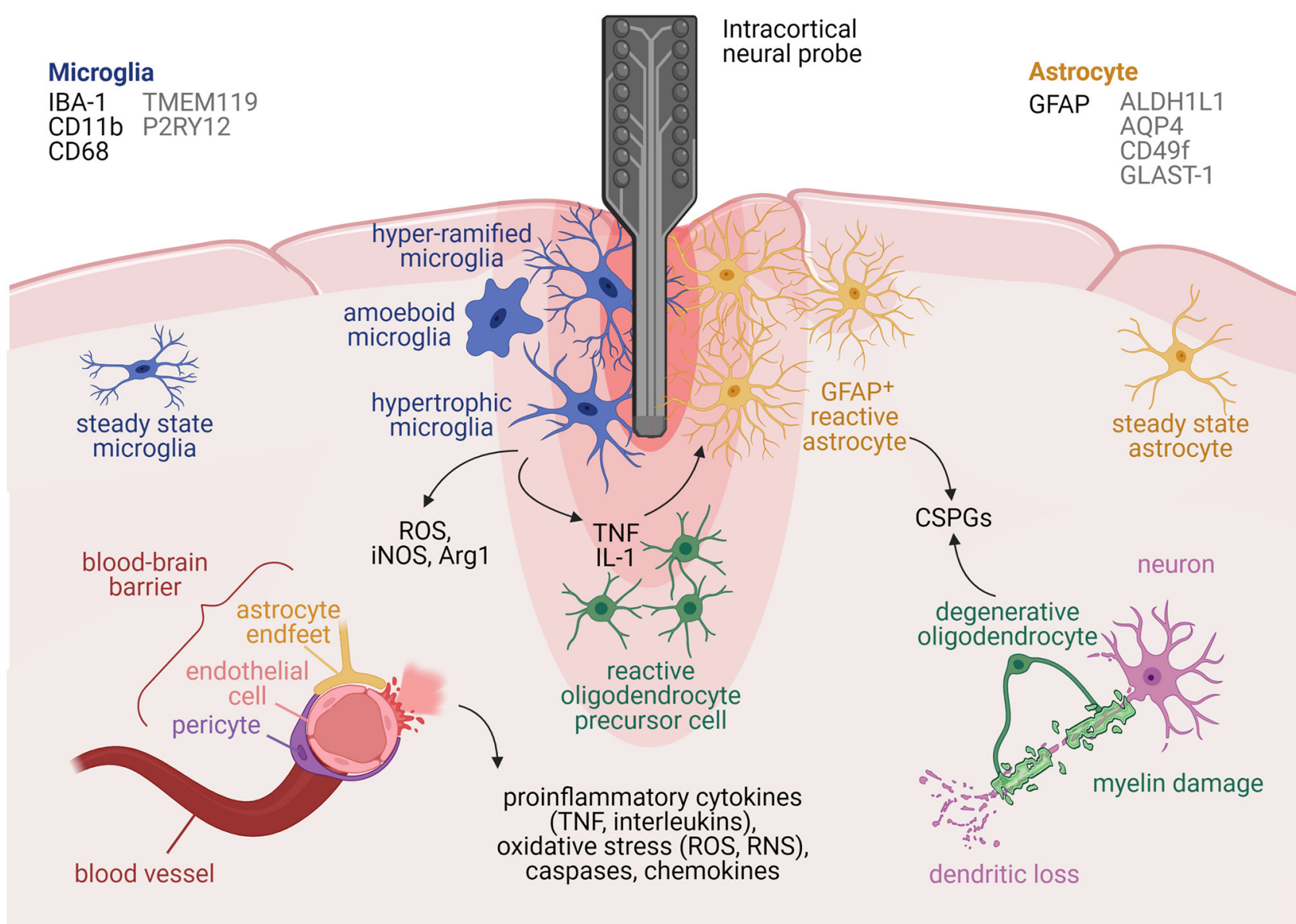

Pericyte

NG2

PDGFR- $\beta$

Blood-brain barrier
integrity
IgG
Evans Blue

Evans Blue

\section{Oligodendrocyte precursor cell \\ NG2}

Oligodendrocyte

CNP, CC1 TMEM10 Olig2, MBP

\section{APPROACHES TO STUDY GLIAL HETEROGENEITY}

\section{Gene expression analysis}

Single-cell RNA-sequencing

Spatial transcriptomics

\section{Protein marker analysis}

Multiplexed immunohistochemistry

\section{Morphological analysis}

\section{Real-time analysis}

Electrophysiology

Widefield, confocal or electron microscopy Two-photon microscopy Multiplexed mass cytometry Cell morphology \& dynamics

FIGURE 1 | Summary of acute glial responses to implantation of neural microprobes in the mammalian brain and proposed approaches to study glial heterogeneity. Common markers (black) and additional markers (gray) for histological analyses of each glial type and blood-brain barrier integrity around the implants are listed. Black arrows indicate secreted molecules. Created with BioRender.com. 
unlikely lead to only destructive outcomes. Conditional ablation of astrocytes after CCI, stab or crush injuries augmented lesion formation, demyelination and death of neurons and oligodendrocytes (Faulkner et al., 2004; Myer et al., 2006). The multifaceted roles of astrocytes suggest they are also vital promoters of repair.

Elucidating long-term changes in astrocytic FBR and scarring at neuroprosthetic implantation sites requires an understanding of astrocyte heterogeneity. Astrocytic diversity is well-described in healthy, developing and diseased CNS (Khakh and Sofroniew, 2015; Chai et al., 2017; Lanjakornsiripan et al., 2018; Clavreul et al., 2019; Pestana et al., 2020) and cannot be represented by the GFAP marker. Markers for homeostatic astrocytes include aldehyde dehydrogenase 1 family member L1 (ALDH1L1) (Cahoy et al., 2008), glutamate aspartate transporter 1 (GLAST1, also known as excitatory amino acid transporter 1, EAAT-1) (Hurwitz et al., 1993) and aquaporin-4 (AQP4) found in astrocytic end feet (Yoneda et al., 2001) (Figure 1). A newly described population of human induced pluripotent stem cells-derived, proinflammatory cytokine-stimulated reactive astrocytes specifically upregulate CD49f (Barbar et al., 2020). From gray matter protoplasmic astrocytes to white matter fibrous astrocytes, diverse astrocytic morphologies in the healthy and diseased brain are well-documented (Zhang and Barres, 2010; Molofsky et al., 2012; Bardehle et al., 2013; Bayraktar et al., 2014). Immunohistochemical analyses to date however exclude the heterogeneity of astrocytes surrounding an implant.

\section{IMPACT OF NEUROPROSTHETICS ON OLIGODENDROCYTES AND THEIR PROGENITORS}

Differentiation of OPCs, also known as NG2-glia, gives rise to oligodendrocytes that produce and maintain myelin sheaths, which provide neurotrophic support and optimize brain electrical signaling (Bradl and Lassmann, 2010; Nave and Werner, 2014). OPCs and newly derived oligodendrocytes are essential for remyelination and CNS repair following demyelinating diseases or brain injury (Young et al., 2013; Bechler et al., 2015). Immunohistochemical analyses of oligodendrocytes at neurointerfaces have involved markers including 2', $3^{\prime}$-Cyclic-nucleotide $3^{\prime}$-phosphodiesterase (CNP) (Chen et al., 2021) and CC1 (a monoclonal antibody against adenomatous polyposis coli) for mature oligodendrocytes, oligodendrocyte transcription factor 2 (Olig2) for immature oligodendrocytes, and myelin basic protein (MBP) for myelinating oligodendrocytes (Wellman et al., 2018, 2019) (Figure 1). TMEM10, a type 1 transmembrane glycoprotein, was recently verified to be specific for mammalian CNS myelin (Golan et al., 2008; de Faria et al., 2019).

With limited antioxidant capacity and high iron content, oligodendrocytes are sensitive to elevated ROS and reactive nitrogen species (RNS) levels arising from glial response to acute implantation injury and FBR (Smith et al., 1999). Similar to $\mathrm{GFAP}^{+}$astrocytes, OPCs and oligodendrocytes release axonal growth-inhibiting CSPGs including NG2 and myelinassociated glycoprotein (Fawcett and Asher, 1999). Studies on passive multi-channel, four-shank "Michigan" MEAs in murine visual cortex revealed acute oligodendrocyte injury and degeneration, myelin degradation, and reactive swarming of OPCs toward the implant within $12 \mathrm{~h}$ (Wellman and Kozai, 2018; Chen et al., 2021). Severe reduction of electrophysiological recording quality from neurons at various tissue depths and observations of decreased neuronal firing in a mouse model of demyelination highlighted the importance of myelin integrity for microelectrode function (Wellman et al., 2020). A clearer picture of the renewal, maturation and function of various oligodendroglia at implantation sites will allow to determine the degree of cohesiveness at the brain-machine interface.

\section{IMPACT OF NEUROPROSTHETICS ON BLOOD-BRAIN BARRIER INTEGRITY}

A breach of the blood-brain barrier (BBB) is inevitable during implantation for microelectrodes to reach the neurons. The $\mathrm{BBB}$ is composed of endothelial cells, pericytes, and astrocytes, forming the neurovascular unit together with surrounding microglia and neurons (Sweeney et al., 2016; Bennett et al., 2019). Cerebrovascular endothelial cells are seamlessly joined by active protein complexes known as tight and adherens junctions (Tietz and Engelhardt, 2015). Pericytes also regulate BBB permeability and are involved in neuroinflammatory response, clearance of toxic metabolites and promotion of angiogenesis (Hill et al., 2014). Pericytes are typically identified by the colocalization of NG2 and platelet-derived growth factor receptor beta (PDGFR- $\beta$ ) markers (to differentiate them from NG2 ${ }^{+}$OPCs) (Wellman et al., 2019) (Figure 1). Vasculature integrity is commonly assessed by histological detection of immunoglobulin G leakage or Evans blue staining for plasma membrane damage (Nolta et al., 2015; Falcone et al., 2019) (Figure 1). Decreased expression of junctional proteins in the compromised $\mathrm{BBB}$ promotes neuroinflammation through higher expression of proinflammatory cytokines and chemokines and increased infiltration of peripheral immune cells (Marchetti and Engelhardt, 2020). BBB leakiness, astrogliosis and neuronal death in brain tissue surrounding the implanted device were shown to reduce the number of measurable electrophysiological responses of single neurons and degrade the overall recording performance of the biosensor (Nolta et al., 2015). High-speed pneumatic intracortical insertion of Utah MEA in rat cortex has led to down-regulation of endothelial tight and adherens junction protein markers, and correlated with increased oxidative stress and elevated inflammation levels indicated by upregulation of caspases, chemokines, interleukins and TNF (Bennett et al., 2018, 2019) (Figure 1). Notably, BBB release of ROS, RNS, and proinflammatory cytokines and chemokines likely promote microglial and astrocyte reactivity, and loss of neurons and oligodendrocytes. 


\section{CURRENT APPROACHES TO STUDY GLIAL HETEROGENEITY}

Single-cell transcriptomic technologies that simultaneously quantify hundreds or thousands of expressed genes of individual cells in a given population have unmasked heterogeneous cellular identities and developmental trajectories, and revealed biomarker information (Aldridge and Teichmann, 2020). These powerful methods provide unique insights into health and disease in contrast to bulk transcriptomic and classical histological analyses. Single-cell RNA-sequencing and spatial transcriptomic approaches have shown that glia isolated from healthy and disease-associated brain regions respond across broad cellular states for microglia (Tay et al., 2018; Hammond et al., 2019; Jordão et al., 2019; Li et al., 2019; Masuda et al., 2019), astrocytes (Cahoy et al., 2008; Zamanian et al., 2012; Boisvert et al., 2018; Bradley et al., 2019; Batiuk et al., 2020; Bayraktar et al., 2020; Das et al., 2020), and oligodendrocytes (Jäkel et al., 2019; Spitzer et al., 2019; Floriddia et al., 2020). Advances in single-cell proteomics have also enabled the highthroughput investigation of key biological questions involving protein binding, modifications, and degradation, that cannot be assessed at the transcriptomic level (Slavov, 2020). Multiplexed mass cytometry and multiplexed immunohistochemistry have unveiled regional and pathology-dependent heterogeneity of human peripheral myeloid cells, microglia and astrocytes (Böttcher et al., 2019; Park et al., 2019). Furthermore, multiplexed immunohistochemistry, electron microscopy and in vivo two-photon imaging techniques are increasingly applied to study acute and chronic oligodendrocyte and OPC reactivity after microprobe implantation (Bogoslovsky et al., 2018; Michelson et al., 2018; Wellman and Kozai, 2018; Chen et al., 2021) (Figure 1). Clearly, advancing MEA technology requires a comprehensive examination of glial responses at neurointerfaces by integrating quantitative single-cell multi-omic analyses with assessments of cell morphology and dynamics, and electrophysiological recordings, as has been recently demonstrated in neurons (Cadwell et al., 2016).

\section{DISCUSSION}

Implantation of single-shank or multi-shank MEAs will inevitably trigger changes in glia and the $\mathrm{BBB}$ due to acute tissue trauma and FBR. Microglia surrounding the lesion will immediately undergo significant state changes to limit physical damage through microgliosis, phagocytosis of dying cells and debris, and release of proinflammatory cytokines and stress-induced molecules. Microglial reactivity likely elevates

\section{REFERENCES}

Aldridge, S., and Teichmann, S. A. (2020). Single cell transcriptomics comes of age. Nat. Commun. 11:4307. doi: 10.1038/s41467-020-18158-5

Alliot, F., Godin, I., and Pessac, B. (1999). Microglia derive from progenitors, originating from the yolk sac, and which proliferate in the brain. Brain Res. Dev. Brain Res. 117, 145-152. doi: 10.1016/S0165-3806(99)00113-3 the population of reactive astrocytes, which could lead to extensive unwanted glial scarring. In concert with astrocytes, degenerative oligodendrocytes also secrete growth-inhibiting extracellular matrix components, and result in electrode encapsulation and dysfunction. Loss of BBB homeostasis also exacerbates proinflammatory responses of microglia and astrocytes to favor neuronal and myelin loss. CNS repair however necessitates acute inflammatory events contributed by neuroprotective subpopulations of non-neuronal brain cells. To harness the endogenous, neuro-regenerative properties of glia and promote electrode biocompatibility and longevity (Gulino et al., 2019), we propose to investigate contextdependent glial responses at brain-machine interfaces using combinatorial approaches in addition to immunohistochemical assays of protein markers. Probe fabrication breakthroughs in material, size and geometry have limited implantation trauma and reduced probe encapsulation (Patel et al., 2016; Luan et al., 2017; Rivnay et al., 2017). Devices coated with dexamethasone to alleviate neuroinflammation (Kozai et al., 2016; Boehler et al., 2017), or laminin to restrict glial reactivity at implantation sites (He et al., 2006), have shown great promise. A deeper molecular understanding of diverse glial responses at neurointerfaces will identify further candidates for promoting neuroprosthetics development.

\section{AUTHOR CONTRIBUTIONS}

OB wrote the first draft of the manuscript and designed the figure. TLT supervised the project and extensively revised the manuscript. All authors contributed to the article and approved the submitted version.

\section{FUNDING}

OB received the Erasmus+ Scholarship (Joint Master in Neuroscience). TLT was supported by the Klaus Tschira Boost Fund (KT-10), FRIAS Junior Fellowship, University of Freiburg Research Innovation Fund, Wissenschaftliche Gesellschaft Freiburg (Helmut Holzer Prize), German Research Foundation (EXC 1086), and Ministry of Economics, Science and Arts of Baden-Württemberg (Sustainability Programme for Projects of the Excellence Initiative II).

\section{ACKNOWLEDGMENTS}

The authors thank C. Böhler and K. Sharma (IMTEK, Freiburg) for feedback. 
Barz, F., Livi, A., Lanzilotto, M., Maranesi, M., Bonini, L., Paul, O., et al. (2017). Versatile, modular 3D microelectrode arrays for neuronal ensemble recordings: from design to fabrication, assembly, and functional validation in non-human primates. J. Neural Eng. 14:036010. doi: 10.1088/1741-2552/aa5a90

Batiuk, M. Y., Martirosyan, A., Wahis, J., de Vin, F., Marneffe, C., Kusserow, C., et al. (2020). Identification of region-specific astrocyte subtypes at single cell resolution. Nat. Commun. 11:1220. doi: 10.1038/s41467-019-14198-8

Bayraktar, O. A., Bartels, T., Holmqvist, S., Kleshchevnikov, V., Martirosyan, A., Polioudakis, D., et al. (2020). Astrocyte layers in the mammalian cerebral cortex revealed by a single-cell in situ transcriptomic map. Nat. Neurosci. 23, 500-509. doi: 10.1038/s41593-020-0602-1

Bayraktar, O. A., Fuentealba, L. C., Alvarez-Buylla, A., and Rowitch, D. H. (2014). Astrocyte development and heterogeneity. Cold Spring Harb. Perspect. Biol. 7:a020362. doi: 10.1101/cshperspect.a020362

Bechler, M. E., Byrne, L., and Ffrench-Constant, C. (2015). CNS myelin sheath lengths are an intrinsic property of oligodendrocytes. Curr. Biol. 25, 2411-2416. doi: 10.1016/j.cub.2015.07.056

Bellver-Landete, V., Bretheau, F., Mailhot, B., Vallières, N., Lessard, M., Janelle, M.-E., et al. (2019). Microglia are an essential component of the neuroprotective scar that forms after spinal cord injury. Nat. Commun. 10:518. doi: 10.1038/s41467-019-08446-0

Bennett, C., Mohammed, F., Álvarez-Ciara, A., Nguyen, M. A., Dietrich, W. D., Rajguru, S. M., et al. (2019). Neuroinflammation, oxidative stress, and bloodbrain barrier $(\mathrm{BBB})$ disruption in acute Utah electrode array implants and the effect of deferoxamine as an iron chelator on acute foreign body response. Biomaterials 188, 144-159. doi: 10.1016/j.biomaterials.2018.09.040

Bennett, C., Samikkannu, M., Mohammed, F., Dietrich, W. D., Rajguru, S. M., and Prasad, A. (2018). Blood brain barrier (BBB)-disruption in intracortical silicon microelectrode implants. Biomaterials 164, 1-10. doi: 10.1016/j.biomaterials.2018.02.036

Bennett, M. L., Bennett, F. C., Liddelow, S. A., Ajami, B., Zamanian, J. L., Fernhoff, N. B., et al. (2016). New tools for studying microglia in the mouse and human CNS. Proc. Natl. Acad. Sci. U. S. A. 113, E1738-1746. doi: $10.1073 /$ pnas. 1525528113

Boehler, C., Carli, S., Fadiga, L., Stieglitz, T., and Asplund, M. (2020). Tutorial: guidelines for standardized performance tests for electrodes intended for neural interfaces and bioelectronics. Nat. Protoc. 15, 3557-3578. doi: 10.1038/s41596-020-0389-2

Boehler, C., Kleber, C., Martini, N., Xie, Y., Dryg, I., Stieglitz, T., et al. (2017). Actively controlled release of Dexamethasone from neural microelectrodes in a chronic in vivo study. Biomaterials 129, 176-187. doi: 10.1016/j.biomaterials.2017.03.019

Bogoslovsky, T., Bernstock, J. D., Bull, G., Gouty, S., Cox, B. M., Hallenbeck, J. M., et al. (2018). Development of a systems-based in situ multiplex biomarker screening approach for the assessment of immunopathology and neural tissue plasticity in male rats after traumatic brain injury. J. Neurosci. Res. 96, 487-500. doi: 10.1002/jnr.24054

Boisvert, M. M., Erikson, G. A., Shokhirev, M. N., and Allen, N. J. (2018). The aging astrocyte transcriptome from multiple regions of the mouse brain. Cell Rep. 22, 269-285. doi: 10.1016/j.celrep.2017.12.039

Böttcher, C., Schlickeiser, S., Sneeboer, M. A. M., Kunkel, D., Knop, A., Paza, E., et al. (2019). Human microglia regional heterogeneity and phenotypes determined by multiplexed single-cell mass cytometry. Nat. Neurosci. 22, 78-90. doi: 10.1038/s41593-018-0290-2

Bradl, M., and Lassmann, H. (2010). Oligodendrocytes: biology and pathology. Acta Neuropathol. 119, 37-53. doi: 10.1007/s00401-009-0601-5

Bradley, R. A., Shireman, J., McFalls, C., Choi, J., Canfield, S. G., Dong, Y., et al. (2019). Regionally specified human pluripotent stem cell-derived astrocytes exhibit different molecular signatures and functional properties. Development 146:dev170910. doi: 10.1242/dev.170910

Budoff, S. A., Yano, K. M., de Mesquita, F. C., Doerl, J. G., de Santana, M. B., Nascimento, M. S. L., et al. (2019). Astrocytic response to acutelyand chronically-implanted microelectrode arrays in the marmoset (Callithrix jacchus) brain. Brain Sci. 9:19. doi: 10.3390/brainsci9020019

Butovsky, O., Jedrychowski, M. P., Moore, C. S., Cialic, R., Lanser, A. J., Gabriely, G., et al. (2014). Identification of a unique TGF- $\beta$-dependent molecular and functional signature in microglia. Nat. Neurosci. 17, 131-143. doi: $10.1038 / \mathrm{nn} .3599$
Cadwell, C. R., Palasantza, A., Jiang, X., Berens, P., Deng, Q., Yilmaz, M., et al. (2016). Electrophysiological, transcriptomic and morphologic profiling of single neurons using Patch-seq. Nat. Biotechnol. 34, 199-203. doi: $10.1038 /$ nbt.3445

Cahoy, J. D., Emery, B., Kaushal, A., Foo, L. C., Zamanian, J. L., Christopherson, K. S., et al. (2008). A transcriptome database for astrocytes, neurons, and oligodendrocytes: a new resource for understanding brain development and function. J. Neurosci. 28, 264-278. doi: 10.1523/JNEUROSCI.4178-07.2008

Chai, H., Diaz-Castro, B., Shigetomi, E., Monte, E., Octeau, J. C., Yu, X., et al. (2017). Neural circuit-specialized astrocytes: transcriptomic, proteomic, morphological, and functional evidence. Neuron 95, 531-549.e9. doi: 10.1016/j.neuron.2017.06.029

Chen, K., Wellman, S. M., Yaxiaer, Y., Eles, J. R., and Kozai, T. D. (2021). In vivo spatiotemporal patterns of oligodendrocyte and myelin damage at the neural electrode interface. Biomaterials 268:120526. doi: 10.1016/j.biomaterials.2020.120526

Clavreul, S., Abdeladim, L., Hernández-Garzón, E., Niculescu, D., Durand, J., Ieng, S.-H., et al. (2019). Cortical astrocytes develop in a plastic manner at both clonal and cellular levels. Nat. Commun. 10:4884. doi: 10.1038/s41467-019-1 2791-5

Das, S., Li, Z., Noori, A., Hyman, B. T., and Serrano-Pozo, A. (2020). Meta-analysis of mouse transcriptomic studies supports a context-dependent astrocyte reaction in acute CNS injury versus neurodegeneration. J. Neuroinflammation 17:227. doi: 10.1186/s12974-020-01898-y

de Faria, O., Dhaunchak, A. S., Kamen, Y., Roth, A. D., Kuhlmann, T., Colman, D. R., et al. (2019). TMEM10 promotes oligodendrocyte differentiation and is expressed by oligodendrocytes in human remyelinating multiple sclerosis plaques. Sci. Rep. 9:3606. doi: 10.1038/s41598-019-40342-x

Ereifej, E. S., Rial, G. M., Hermann, J. K., Smith, C. S., Meade, S. M., Rayyan, J. M., et al. (2018). Implantation of neural probes in the brain elicits oxidative stress. Front. Bioeng. Biotechnol. 6:9. doi: 10.3389/fbioe.2018.00009

Falcone, J. D., Sohal, H. S., Kyriakides, T. R., and Bellamkonda, R. V. (2019). The impact of modulating the blood-brain barrier on the electrophysiological and histological outcomes of intracortical electrodes. J. Neural Eng. 16:046005. doi: 10.1088/1741-2552/ablef9

Faulkner, J. R., Herrmann, J. E., Woo, M. J., Tansey, K. E., Doan, N. B., and Sofroniew, M. V. (2004). Reactive astrocytes protect tissue and preserve function after spinal cord injury. J. Neurosci. 24, 2143-2155. doi: 10.1523/JNEUROSCI.3547-03.2004

Fawcett, J. W., and Asher, R. A. (1999). The glial scar and central nervous system repair. Brain Res. Bull. 49, 377-391. doi: 10.1016/S0361-9230(99)00072-6

Floriddia, E. M., Lourenço, T., Zhang, S., van Bruggen, D., Hilscher, M. M., Kukanja, P., et al. (2020). Distinct oligodendrocyte populations have spatial preference and different responses to spinal cord injury. Nat. Commun. 11:5860. doi: 10.1038/s41467-020-19453-x

Galtrey, C. M., and Fawcett, J. W. (2007). The role of chondroitin sulfate proteoglycans in regeneration and plasticity in the central nervous system. Brain Res. Rev. 54, 1-18. doi: 10.1016/j.brainresrev.2006.09.006

Gerbella, M., Borra, E., Pothof, F., Lanzilotto, M., Livi, A., Fogassi, L., et al. (2021). Histological assessment of a chronically implanted cylindricallyshaped, polymer-based neural probe in the monkey. J. Neural Eng. 18:024001. doi: 10.1088/1741-2552/abdd11

Ginhoux, F., Greter, M., Leboeuf, M., Nandi, S., See, P., Gokhan, S., et al. (2010). Fate mapping analysis reveals that adult microglia derive from primitive macrophages. Science 330, 841-845. doi: 10.1126/science.1194637

Ginhoux, F., Lim, S., Hoeffel, G., Low, D., and Huber, T. (2013). Origin and differentiation of microglia. Front. Cell. Neurosci. 7:45. doi: $10.3389 /$ fncel.2013.00045

Golan, N., Adamsky, K., Kartvelishvily, E., Brockschnieder, D., Möbius, W., Spiegel, I., et al. (2008). Identification of Tmem10/Opalin as an oligodendrocyte enriched gene using expression profiling combined with genetic cell ablation. Glia 56, 1176-1186. doi: 10.1002/glia.20688

Gulino, M., Kim, D., Pan,é, S., Santos, S. D., and Pêgo, A. P. (2019). Tissue response to neural implants: the use of model systems toward new design solutions of implantable microelectrodes. Front. Neurosci. 13:689. doi: 10.3389/fnins.2019.00689

Hammond, T. R., Dufort, C., Dissing-Olesen, L., Giera, S., Young, A., Wysoker, A., et al. (2019). Single-cell RNA sequencing of microglia throughout the mouse 
lifespan and in the injured brain reveals complex cell-state changes. Immunity 50, 253-271.e6. doi: 10.1016/j.immuni.2018.11.004

He, F., Lycke, R., Ganji, M., Xie, C., and Luan, L. (2020). Ultraflexible neural electrodes for long-lasting intracortical recording. iScience 23:101387. doi: 10.1016/j.isci.2020.101387

He, W., McConnell, G. C., and Bellamkonda, R. V. (2006). Nanoscale laminin coating modulates cortical scarring response around implanted silicon microelectrode arrays. J. Neural Eng. 3, 316-326. doi: 10.1088/1741-2560/3/4/009

Hill, J., Rom, S., Ramirez, S. H., and Persidsky, Y. (2014). Emerging roles of pericytes in the regulation of the neurovascular unit in health and disease. $J$. Neuroimmune. Pharmacol. 9, 591-605. doi: 10.1007/s11481-014-9557-x

Huang, S.-H., Shmoel, N., Jankowski, M. M., Erez, H., Sharon, A., Abu-Salah, W., et al. (2020). Immunohistological and ultrastructural study of the inflammatory response to perforated polyimide cortical implants: mechanisms underlying deterioration of electrophysiological recording quality. Front. Neurosci. 14:926. doi: 10.3389/fnins.2020.00926

Hurwitz, A. A., Berman, J. W., Rashbaum, W. K., and Lyman, W. D. (1993). Human fetal astrocytes induce the expression of blood-brain barrier specific proteins by autologous endothelial cells. Brain Res. 625, 238-243. doi: 10.1016/0006-8993(93)91064-Y

Jäkel, S., Agirre, E., Mendanha Falcão, A., van Bruggen, D., Lee, K. W., Knuesel, I., et al. (2019). Altered human oligodendrocyte heterogeneity in multiple sclerosis. Nature 566, 543-547. doi: 10.1038/s41586-019-0903-2

Jordão, M. J. C., Sankowski, R., Brendecke, S. M., Locatelli, G., Tai, Y. H., Tay, T. L., et al. (2019). Single-cell profiling identifies myeloid cell subsets with distinct fates during neuroinflammation. Science 363:eaat7554. doi: 10.1126/science.aat7554

Khakh, B. S., and Sofroniew, M. V. (2015). Diversity of astrocyte functions and phenotypes in neural circuits. Nat. Neurosci. 18, 942-952. doi: 10.1038/nn.4043

Kozai, T. D. Y., Jaquins-Gerstl, A. S., Vazquez, A. L., Michael, A. C., and Cui, X. T. (2015). Brain tissue responses to neural implants impact signal sensitivity and intervention strategies. ACS Chem. Neurosci. 6, 48-67. doi: 10.1021/cn50 $0256 \mathrm{e}$

Kozai, T. D. Y., Jaquins-Gerstl, A. S., Vazquez, A. L., Michael, A. C., and Cui, X. T. (2016). Dexamethasone retrodialysis attenuates microglial response to implanted probes in vivo. Biomaterials 87, 157-169. doi: 10.1016/j.biomaterials.2016.02.013

Kozai, T. D. Y., Vazquez, A. L., Weaver, C. L., Kim, S.-G., and Cui, X. T. (2012). In vivo two-photon microscopy reveals immediate microglial reaction to implantation of microelectrode through extension of processes. J. Neural Eng. 9:066001. doi: 10.1088/1741-2560/9/6/066001

Lanjakornsiripan, D., Pior, B.-J., Kawaguchi, D., Furutachi, S., Tahara, T., Katsuyama, Y., et al. (2018). Layer-specific morphological and molecular differences in neocortical astrocytes and their dependence on neuronal layers. Nat. Commun. 9:1623. doi: 10.1038/s41467-018-03940-3

Lau, L. W., Keough, M. B., Haylock-Jacobs, S., Cua, R., Döring, A., Sloka, S., et al. (2012). Chondroitin sulfate proteoglycans in demyelinated lesions impair remyelination. Ann. Neurol. 72, 419-432. doi: 10.1002/ana.23599

Lewis, T. (2020). Elon Musk's Pig-Brain Implant Is Still a Long Way from "Solving Paralysis". Available online at: https://www.scientificamerican.com/article/ elon-musks-pig-brain-implant-is-still-a-long-way-from-solving-paralysis/ (accessed January 22, 2021).

Li, Q., Cheng, Z., Zhou, L., Darmanis, S., Neff, N. F., Okamoto, J., et al. (2019). Developmental heterogeneity of microglia and brain myeloid cells revealed by deep single-cell RNA sequencing. Neuron 101, 207-223.e10. doi: 10.1016/j.neuron.2018.12.006

Liddelow, S. A., and Barres, B. A. (2017). Reactive astrocytes: production, function, and therapeutic potential. Immunity 46, 957-967. doi: 10.1016/j.immuni.2017.06.006

Liddelow, S. A., Guttenplan, K. A., Clarke, L. E., Bennett, F. C., Bohlen, C. J., Schirmer, L., et al. (2017). Neurotoxic reactive astrocytes are induced by activated microglia. Nature 541, 481-487. doi: 10.1038/nature21029

Lind, G., Linsmeier, C. E., and Schouenborg, J. (2013). The density difference between tissue and neural probes is a key factor for glial scarring. Sci. Rep. 3:2942. doi: 10.1038/srep02942

Lo, M.-C., Wang, S., Singh, S., Damodaran, V. B., Ahmed, I., Coffey, K., et al. (2018). Evaluating the in vivo glial response to miniaturized parylene cortical probes coated with an ultra-fast degrading polymer to aid insertion. J. Neural Eng. 15:036002. doi: 10.1088/1741-2552/aa9fad

Luan, L., Wei, X., Zhao, Z., Siegel, J. J., Potnis, O., Tuppen, C. A., et al. (2017). Ultraflexible nanoelectronic probes form reliable, glial scar-free neural integration. Sci. Adv. 3:e1601966. doi: 10.1126/sciadv.1601966

Madathil, S. K., Wilfred, B. S., Urankar, S. E., Yang, W., Leung, L. Y., Gilsdorf, J. S., et al. (2018). Early microglial activation following closed-head concussive injury is dominated by pro-inflammatory M-1 type. Front. Neurol. 9:964. doi: 10.3389/fneur.2018.00964

Marchetti, L., and Engelhardt, B. (2020). Immune cell trafficking across the bloodbrain barrier in the absence and presence of neuroinflammation. Vasc. Biol. 2, H1-H18. doi: 10.1530/VB-19-0033

Masuda, T., Sankowski, R., Staszewski, O., Böttcher, C., and Amann, L., Sagar, et al. (2019). Spatial and temporal heterogeneity of mouse and human microglia at single-cell resolution. Nature 566, 388-392. doi: 10.1038/s41586-019-0924-x

Masuda, T., Sankowski, R., Staszewski, O., and Prinz, M. (2020). Microglia heterogeneity in the single-cell era. Cell Rep. 30, 1271-1281. doi: 10.1016/j.celrep.2020.01.010

Matsui, F., Kawashima, S., Shuo, T., Yamauchi, S., Tokita, Y., Aono, S., et al. (2002). Transient expression of juvenile-type neurocan by reactive astrocytes in adult rat brains injured by kainate-induced seizures as well as surgical incision. Neuroscience 112, 773-781. doi: 10.1016/S0306-4522(02)00136-7

McConnell, G. C., Rees, H. D., Levey, A. I., Gutekunst, C.-A., Gross, R. E., and Bellamkonda, R. V. (2009). Implanted neural electrodes cause chronic, local inflammation that is correlated with local neurodegeneration. J. Neural Eng. 6:056003. doi: 10.1088/1741-2560/6/5/056003

McCreery, D., Cogan, S., Kane, S., and Pikov, V. (2016). Correlations between histology and neuronal activity recorded by microelectrodes implanted chronically in the cerebral cortex. J. Neural Eng. 13:036012. doi: 10.1088/1741-2560/13/3/036012

McKeon, R. J., Jurynec, M. J., and Buck, C. R. (1999). The chondroitin sulfate proteoglycans neurocan and phosphacan are expressed by reactive astrocytes in the chronic CNS glial scar. J. Neurosci. 19, 10778-10788. doi: 10.1523/JNEUROSCI.19-24-10778.1999

McKeon, R. J., Schreiber, R. C., Rudge, J. S., and Silver, J. (1991). Reduction of neurite outgrowth in a model of glial scarring following CNS injury is correlated with the expression of inhibitory molecules on reactive astrocytes. J. Neurosci. 11, 3398-3411. doi: 10.1523/JNEUROSCI.11-11-03398.1991

Michelson, N. J., Vazquez, A. L., Eles, J. R., Salatino, J. W., Purcell, E. K., Williams, J. J., et al. (2018). Multi-scale, multi-modal analysis uncovers complex relationship at the brain tissue-implant neural interface: new emphasis on the biological interface. J. Neural Eng. 15:033001. doi: 10.1088/1741-2552/aa9dae

Molofsky, A. V., Krencik, R., Krenick, R., Ullian, E. M., Ullian, E., Tsai, H., et al. (2012). Astrocytes and disease: a neurodevelopmental perspective. Genes Dev. 26, 891-907. doi: 10.1101/gad.188326.112

Myer, D. J., Gurkoff, G. G., Lee, S. M., Hovda, D. A., and Sofroniew, M. V. (2006). Essential protective roles of reactive astrocytes in traumatic brain injury. Brain 129, 2761-2772. doi: 10.1093/brain/awl165

Nave, K.-A., and Werner, H. B. (2014). Myelination of the nervous system: mechanisms and functions. Annu. Rev. Cell Dev. Biol. 30, 503-533. doi: 10.1146/annurev-cellbio-100913-013101

Nolta, N. F., Christensen, M. B., Crane, P. D., Skousen, J. L., and Tresco, P. A. (2015). BBB leakage, astrogliosis, and tissue loss correlate with silicon microelectrode array recording performance. Biomaterials 53, 753-762. doi: 10.1016/j.biomaterials.2015.02.081

Park, C., Ponath, G., Levine-Ritterman, M., Bull, E., Swanson, E. C., De Jager, P. L., et al. (2019). The landscape of myeloid and astrocyte phenotypes in acute multiple sclerosis lesions. Acta Neuropathol. Commun. 7:130. doi: 10.1186/s40478-019-0779-2

Patel, P. R., Zhang, H., Robins, M. T., Nofar, J. B., Marshall, S. P., Kobylarek, M. J., et al. (2016). Chronic in vivo stability assessment of carbon fiber microelectrode arrays. J. Neural Eng. 13:066002. doi: 10.1088/1741-2560/13/6/066002

Pestana, F., Edwards-Faret, G., Belgard, T. G., Martirosyan, A., and Holt, M. G. (2020). No longer underappreciated: the emerging concept of astrocyte heterogeneity in neuroscience. Brain Sci. 10:168. doi: 10.3390/brainsci10030168

Polikov, V. S., Tresco, P. A., and Reichert, W. M. (2005). Response of brain tissue to chronically implanted neural electrodes. J. Neurosci. Methods 148, 1-18. doi: 10.1016/j.jneumeth.2005.08.015 
Prinz, M., Jung, S., and Priller, J. (2019). Microglia biology: one century of evolving concepts. Cell 179, 292-311. doi: 10.1016/j.cell.2019.08.053

Prodanov, D., and Delbeke, J. (2016). Mechanical and biological interactions of implants with the brain and their impact on implant design. Front. Neurosci. 10:11. doi: 10.3389/fnins.2016.00011

Ritzel, R. M., He, J., Li, Y., Cao, T., Khan, N., Shim, B., et al. (2021). Proton extrusion during oxidative burst in microglia exacerbates pathological acidosis following traumatic brain injury. Glia 69, 746-764. doi: 10.1002/glia.23926

Rivnay, J., Wang, H., Fenno, L., Deisseroth, K., and Malliaras, G. G. (2017). Next-generation probes, particles, and proteins for neural interfacing. Sci. Adv. 3:e1601649. doi: 10.1126/sciadv.1601649

Sawyer, A. J., Tian, W., Saucier-Sawyer, J. K., Rizk, P. J., Saltzman, W. M., Bellamkonda, R. V., et al. (2014). The effect of inflammatory cell-derived MCP1 loss on neuronal survival during chronic neuroinflammation. Biomaterials 35, 6698-6706. doi: 10.1016/j.biomaterials.2014.05.008

Seymour, J. P., and Kipke, D. R. (2007). Neural probe design for reduced tissue encapsulation in CNS. Biomaterials 28, 3594-3607. doi: 10.1016/j.biomaterials.2007.03.024

Slavov, N. (2020). Unpicking the proteome in single cells. Science 367, 512-513. doi: 10.1126/science.aaz6695

Smith, K. J., Kapoor, R., and Felts, P. A. (1999). Demyelination: the role of reactive oxygen and nitrogen species. Brain Pathol. 9, 69-92. doi: 10.1111/j.1750-3639.1999.tb00212.x

Sofroniew, M. V., and Vinters, H. V. (2010). Astrocytes: biology and pathology. Acta Neuropathol. 119, 7-35. doi: 10.1007/s00401-009-0619-8

Spitzer, S. O., Sitnikov, S., Kamen, Y., Evans, K. A., Kronenberg-Versteeg, D., Dietmann, S., et al. (2019). Oligodendrocyte progenitor cells become regionally diverse and heterogeneous with age. Neuron 101, 459-471.e5. doi: 10.1016/j.neuron.2018.12.020

Stratoulias, V., Venero, J. L., Tremblay, M.-Ė., and Joseph, B. (2019). Microglial subtypes: diversity within the microglial community. EMBO J. 38:e101997. doi: 10.15252/embj.2019101997

Sweeney, M. D., Ayyadurai, S., and Zlokovic, B. V. (2016). Pericytes of the neurovascular unit: key functions and signaling pathways. Nat. Neurosci. 19, 771-783. doi: 10.1038/nn.4288

Takmakov, P., Ruda, K., Scott Phillips, K., Isayeva, I. S., Krauthamer, V., and Welle, C. G. (2015). Rapid evaluation of the durability of cortical neural implants using accelerated aging with reactive oxygen species. J. Neural Eng. 12:026003. doi: 10.1088/1741-2560/12/2/026003

Tay, T. L., Dautzenberg, J., Grün, D., and Prinz, M. (2018). Unique microglia recovery population revealed by single-cell RNAseq following neurodegeneration. Acta Neuropathol. Commun. 6:87. doi: 10.1186/s40478-018-0584-3

Tietz, S., and Engelhardt, B. (2015). Brain barriers: crosstalk between complex tight junctions and adherens junctions. J. Cell. Biol. 209, 493-506. doi: $10.1083 /$ jcb.201412147

Turner, J. N., Shain, W., Szarowski, D. H., Andersen, M., Martins, S., Isaacson, M., et al. (1999). Cerebral astrocyte response to micromachined silicon implants. Exp. Neurol. 156, 33-49. doi: 10.1006/exnr.1998.6983
Verdonk, F., Roux, P., Flamant, P., Fiette, L., Bozza, F. A., Simard, S., et al. (2016). Phenotypic clustering: a novel method for microglial morphology analysis. $J$. Neuroinflammation 13:153. doi: 10.1186/s12974-016-0614-7

Verkhratsky, A., and Nedergaard, M. (2018). Physiology of astroglia. Physiol. Rev. 98, 239-389. doi: 10.1152/physrev.00042.2016

Wellman, S. M., Cambi, F., and Kozai, T. D. (2018). The role of oligodendrocytes and their progenitors on neural interface technology: a novel perspective on tissue regeneration and repair. Biomaterials 183, 200-217. doi: 10.1016/j.biomaterials.2018.08.046

Wellman, S. M., Guzman, K., Stieger, K. C., Brink, L. E., Sridhar, S., Dubaniewicz, M. T., et al. (2020). Cuprizone-induced oligodendrocyte loss and demyelination impairs recording performance of chronically implanted neural interfaces. Biomaterials 239:119842. doi: 10.1016/j.biomaterials.2020.1 19842

Wellman, S. M., and Kozai, T. D. Y. (2018). In vivo spatiotemporal dynamics of NG2 glia activity caused by neural electrode implantation. Biomaterials 164, 121-133. doi: 10.1016/j.biomaterials.2018.02.037

Wellman, S. M., Li, L., Yaxiaer, Y., McNamara, I., and Kozai, T. D. Y. (2019). Revealing spatial and temporal patterns of cell death, glial proliferation, and blood-brain barrier dysfunction around implanted intracortical neural interfaces. Front. Neurosci. 13:493. doi: 10.3389/fnins.2019.00493

Yoneda, K., Yamamoto, N., Asai, K., Sobue, K., Fujita, Y., Fujita, M., et al. (2001). Regulation of aquaporin-4 expression in astrocytes. Brain Res. Mol. Brain Res. 89, 94-102. doi: 10.1016/S0169-328X(01)00067-5

Young, K. M., Psachoulia, K., Tripathi, R. B., Dunn, S.-J., Cossell, L., Attwell, D., et al. (2013). Oligodendrocyte dynamics in the healthy adult CNS: evidence for myelin remodeling. Neuron 77, 873-885. doi: 10.1016/j.neuron.2013. 01.006

Zamanian, J. L., Xu, L., Foo, L. C., Nouri, N., Zhou, L., Giffard, R. G., et al. (2012). Genomic analysis of reactive astrogliosis. J. Neurosci. 32, 6391-6410. doi: 10.1523/JNEUROSCI.6221-11.2012

Zhang, Y., and Barres, B. A. (2010). Astrocyte heterogeneity: an underappreciated topic in neurobiology. Curr. Opin. Neurobiol. 20, 588-594. doi: 10.1016/j.conb.2010.06.005

Zhong, Y., and Bellamkonda, R. V. (2007). Dexamethasone-coated neural probes elicit attenuated inflammatory response and neuronal loss compared to uncoated neural probes. Brain Res. 1148, 15-27. doi: 10.1016/j.brainres.2007.02.024

Conflict of Interest: The authors declare that the research was conducted in the absence of any commercial or financial relationships that could be construed as a potential conflict of interest.

Copyright (c) 2021 Bouadi and Tay. This is an open-access article distributed under the terms of the Creative Commons Attribution License (CC BY). The use, distribution or reproduction in other forums is permitted, provided the original author(s) and the copyright owner(s) are credited and that the original publication in this journal is cited, in accordance with accepted academic practice. No use, distribution or reproduction is permitted which does not comply with these terms. 